\title{
Sentence repetition in German-speaking individuals with Down syndrome
}

Bernadette Witecy, Tatjana Tolkmit, Martina Penke

Department of Special Education and Rehabilitation, University of Cologne, Germany https://doi.org/10.36505/ExLing-2020/11/0055/000470

\begin{abstract}
The present study investigated the morphosyntactic abilities of German-speaking individuals with Down syndrome (DS) employing a sentence repetition task. In addition, a nonword repetition task was used to assess verbal short-term memory. The performance of 16 children/adolescents with DS was compared to that of 10 typically developing (TD) children. Group comparisons as well as the inspection of standard scores that were determined based on nonverbal mental age indicated a significant morphosyntactic impairment in most individuals with DS that could neither be solely attributed to the general cognitive delay nor to the observed deficit in verbal verbal short-term memory. Further qualitative results are presented.
\end{abstract}

Keywords: down syndrome, developmental language disorder, morphosyntax, sentence repetition, verbal short-term memory

\section{Introduction}

Individuals with Down syndrome (DS), a neurodevelopmental disorder commonly causing intellectual disability, display noticeable problems in the comprehension and production of syntactic constructions and inflectional morphology (Abbeduto et al. 2007). Verbal short-term memory (VSTM) is another domain where individuals with DS have been found to be significantly impaired (Baddeley, Jarrold 2007) and is discussed as one possible source of the language difficulties in this group (Laws, Bishop 2004). The aim of the present study was to investigate the performance of German-speaking individuals with DS in a standardized sentence repetition (SR) task, commonly used to assess morphosyntactic abilities, and to compare it to that of typically developing (TD) children. Quantitative and qualitative analyses were performed to explore how the results are related to the performance in nonword repetition (NWR), a task that is suitable to assess VSTM in individuals with DS.

\section{Method}

Sixteen monolingual German-speaking children and adolescents with DS ( 9 male), aged 4;6 to 19;0 years ( $M$ 12;4 years), took part in the study. For two of them the parents reported a mild hearing loss. For the remaining participants with DS no permanent hearing loss had been diagnosed. Ten TD children were

ExLing 2020: Proceedings of 11 th International Conference of Experimental Linguistics, 12-14 October 2020, Athens, Greece 
included as controls. Their mean chronological age was 4;11 years (range: 3;11 to $5 ; 7$ years).

Nonverbal mental age (MA) in both groups was assessed using the SON-R 2.5-7 (Tellegen et al. 2007). It ranged from $4 ; 0$ to $6 ; 5$ years $(M 5 ; 1)$ in the individuals with DS and from $4 ; 0$ to $5 ; 3$ years $(M 4 ; 9)$ in the control group (no significant difference, $t(23)=.379, p=.708)$.

VSTM was assessed using a NWR task consisting of 18 nonwords with increasing number of syllables and syllable complexity. The SR task included 15 sentences (length: 6-10 words), six of them semantically meaningful and nine with no meaningful content (e.g.: The clapping chairs sing a cup). Both tasks are part of a standardized test on German language development (SETK 3-5, Grimm et al. 2001). Raw scores (number of correctly repeated nonwords and correctly repeated words out of all sentences, respectively) and standard scores (T-scores), based on the MA of the participants, were calculated according to the test's manual. The maximum obtainable raw score was 18 for NWR and 119 for SR. In addition, results in SR were analysed qualitatively.

\section{Results}

The results of the two groups in NWR and SR are displayed in Table 1. Group comparisons revealed that the TD children performed significantly better than the DS participants in both tasks (comparison of raw scores: NWR: $t(24)=$ $2.319, p=.029$, SR: $t(24)=-6.891, p<.001)$. Performance in NWR and SR was highly correlated in the DS group $(r(13)=.769 ; p=.001)$, but not in the control group $(r(10)=.467 ; p=.173)$.

In almost all participants with DS (13 of 16) standardized T-scores were higher in NWR than in SR. While eight of them performed according to MA expectations in NWR (T-score of 40 or above), only three did so in the SR task. Thus, five participants displayed a dissociation between performance in NWR and in SR.

A comparison of the number of correctly repeated content and function words in the SR task revealed that the participants with DS performed significantly worse on function words compared to content words $(t(15)=$ $8.101, p<.001)$, whereas no such difference between word types could be found in the control group $(t(9)=.059, p=.954)($ see Table 1).

Errors in the SR task were classified as omissions, substitutions (including substitutions of the stem and/or the morphological marking) and additions. Only omissions and substitutions were further analysed. A repeated measures ANOVA with GROUP as between-subjects factor and WORD CLASS (function vs. content word) and ERROR TYPE (omission, substitution) as within-subject factors yielded a significant ERROR TYPE x GROUP interaction $(F(1,24)=5.273$, $p=.031)$. The DS group produced more omissions $(62.9 \%)$ than substitutions (37.1\%). The reverse held for the TD participants $(44.0 \%$ vs. $56.0 \%)$. The three-way interaction between GROUP, WORD CLASS and ERROR TYPE was also 
significant $(F(1,24)=16.461, p<.001)$. This was due to the higher number of omissions of function words in the DS group (see Figure 1). The other effects were not significant $(p>0.5)$.

Table 1. Mean scores for NWR and SR (standard deviations in parentheses). Tscores based on MA in the DS group.

\begin{tabular}{|c|c|c|}
\hline & DS & TD \\
\hline NWR (raw scores) & $6(10.4)$ & $9.5(3.6)$ \\
\hline NWR (T-scores) & $38.8(10.4)$ & $49(15.5)$ \\
\hline SR (raw scores) & $43.7(19.7)$ & $93.2(14.2)$ \\
\hline SR (T-scores) & $33.4(7.1)$ & $55.3(7.1)$ \\
\hline Content words (\% correct) & $47.7 \%(15.8)$ & $80.1 \%(10.5)$ \\
\hline Function words (\% correct) & $27.5 \%(20.5)$ & $80.0 \%(11.0)$ \\
\hline
\end{tabular}

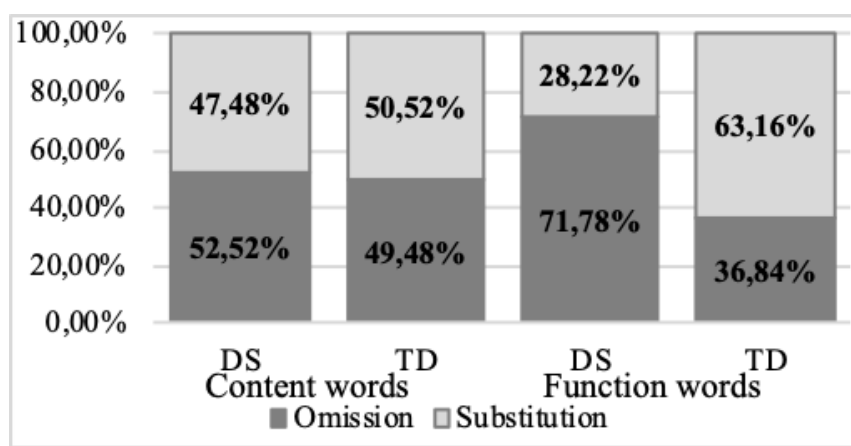

Figure 1. Percentage of omissions and substitutions for each group. Number of errors analyzed: DS: $\mathrm{n}=948$, TD: $\mathrm{n}=258$.

We furthermore examined the nature of the produced utterances in the SR task and graded them into four categories: (1) verbatim responses, (2) imitation with changes, but grammatical with respect to word order, (3) ungrammatical utterances, and (4) one-word-utterances. Whereas the TD children mainly produced grammatical sentences $(89.7 \%)$, in the DS group almost two third of the utterances $(64.8 \%)$ were ungrammatical (Table 2).

Table 2. Results of the syntactic analysis (Number of analysed utterances: DS: $\mathrm{n}=227$, TD: $\mathrm{n}=175$ ).

\begin{tabular}{|c|c|c|c|c|}
\hline & verbatim & correct word order & incorrect word order & single-word \\
\hline DS & $2.6 \%$ & $32.6 \%$ & $59.0 \%$ & $5.7 \%$ \\
\hline TD & $40.0 \%$ & $49.7 \%$ & $10.03 \%$ & $0.0 \%$ \\
\hline
\end{tabular}




\section{Discussion}

The findings indicate a severe morphosyntactic impairment in most of the participants with DS that cannot simply be attributed to the general cognitive delay as most participants performed below MA expectations. In addition, even though results in NWR and SR were correlated in the DS group, the observation that performance in NWR was generally better and often dissociated from performance in SR suggests that the morphosyntactic impairment cannot be solely attributed to a deficit in VSTM. Finally, the higher error-proneness of function words, the observation that function words were omitted rather than substituted and the larger number of ungrammatical utterances in the DS group all point to a grammatical component of the disorder. Our findings support the assumption that morphosyntactic deficits in individuals with DS cannot be reduced to limitations in VSTM (Penke \& Wimmer 2020).

\section{Acknowledgements}

We would like to thank all participants and their families as well as everyone who was involved in data collection and analysis. This research was supported by the German Science Foundation (DFG, grant PE 683/3-1).

\section{References}

Abbeduto, L., Warren, S., Conners, F. 2007. Language development in Down syndrome. Mental Retardation and Developmental Disabilities Research Reviews 13 (3), 247-261.

Baddeley, A., Jarrold, C. 2007. Working memory and Down syndrome. Journal of Intellectual Disability Research 51 (12), 925-931.

Grimm, H., Aktas, M., Frevert, S. 2001. SETK 3-5: Sprachentwicklungstest für dreibis fünfjährige Kinder. Göttingen, Hogrefe.

Laws, G., Bishop, D. 2004. Verbal deficits in Down syndrome and specific language impairment. International Journal of Language \& Communication Disorders 39 (4), 423-451.

Penke, M., Wimmer, E. 2020. Verbal short-term memory and sentence comprehension in German children and adolescents with Down syndrome - beware of the task. First Language 40 (4), 367-389.

Tellegen, P., Laros, J., Petermann, F. 2007. SON-R 2,5-7. Non-verbaler Intelligenztest. Göttingen, Hogrefe. 